\title{
Meta-Atom Loaded Patch Antenna
}

\author{
T. Whittaker ${ }^{1}$, W. Whittow ${ }^{2}$, J. (Yiannis). C. Vardaxoglou ${ }^{3}$ \\ Wolfson School of Mechanical, Electrical and Manufacturing Engineering \\ Loughborough University, Loughborough, UK \\ ${ }^{1}$ T.Whittaker@lboro.ac.uk, ${ }^{2}$ W.G.Whittow@lboro.ac.uk, ${ }^{3}$ J.C.Vardaxoglou@lboro.ac.uk
}

Keywords: Additive Manufacturing, Patch Antenna, Metaatoms, Metamaterial

\begin{abstract}
The design of a meta-atom/metamaterial assisted patch antenna is presented. By utilizing strategically placed meta-atom in the patch antenna design miniaturization benefits can be gained with minimal impact to the total efficiency and the fractional bandwidth.
\end{abstract}

\section{Introduction}

With the advancement of additive manufacturing, integrating $3 \mathrm{D}$ metamaterials into electromagnetic designs is becoming a more viable method of obtaining novel and innovative designs. Metamaterials exhibit material characteristics not normally found in nature which electromagnetic engineers can exploit to improve their designs; for example design miniaturisation [1] and wider bandwidths [2]. This paper presents the concept of utilizing meta-atom technology to design miniaturized antennas. The design of a $3 \mathrm{D}$ printable metamaterial/meta-atom assisted patch antenna is presented to showcase the benefits. The definition of a meta-atom is a metallic or dielectric inclusion having sub-wavelength dimensions; they can be considered as the building blocks of metamaterials. The meta-atoms used in this paper are simple metallic cuboids. As shown in previous literature, metallic inclusions in a dielectric host both increase the overall effective permittivity of the mixture whilst decreasing the effective permeability [3], [4]. These characteristics are exploited in the patch design. Simulation results are used to compare the design of a conventional patch antenna to two

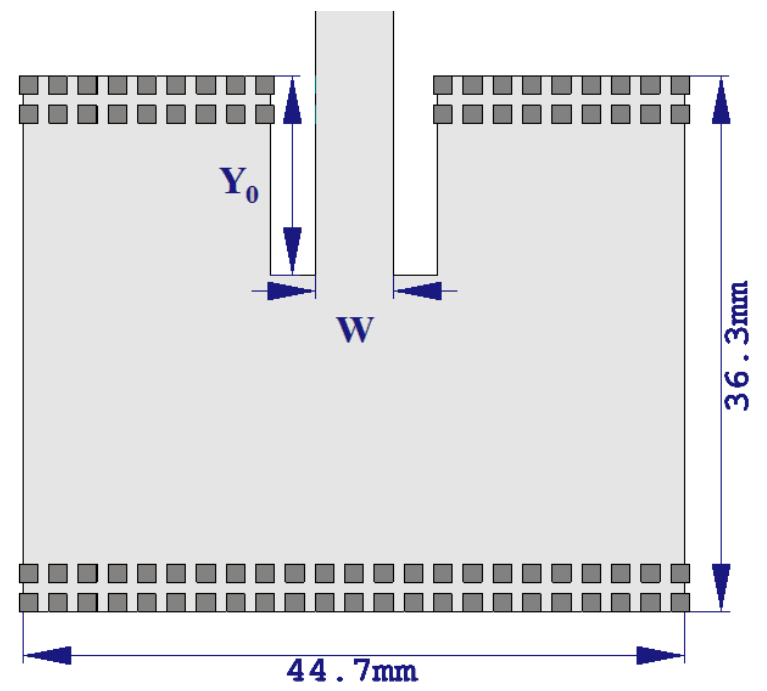

Fig. 1. 2D top view drawing of the patch antenna geometery with the additional cubic meta-atoms in dark grey. metamaterial/meta-atom assisted patch antennas. One patch having an artificial dielectric substrate whilst the other utilises strategically placed meta-atoms underneath the patch's radiating edge. Previously work shows that incorporating dielectric meta-atoms to make an artificial dielectric substrate can provide miniaturisation properties to an antenna [1]. By using a higher volume fraction of metallic meta-atoms the miniaturisation effect can be increased. Also, locating the meta-atoms at critical regions in the design the efficiency and effective bandwidth of the antenna can be largely retained compared to the conventional patch.

\section{Antenna Design}

The geometry of the patch antenna geometry is shown in Fig.1. and the equations used to calculate the dimensions are given by Balanis [5]. The antenna is designed on a $2 \mathrm{~mm}$ thick substrate of PLA (Polylactic acid) which has a relative permittivity of 2.6 and a loss tangent of 0.009 [6]. For a patch antenna designed at $2.5 \mathrm{GHz}$ this gives width of $36.3 \mathrm{~mm}$ and a patch length of $44.7 \mathrm{~mm}$. This antenna will be modified to include cubic meta-atoms along the radiating edges. The aim is to increase the electric coupling at the edges to electrically increase the antenna's length. As the majority of the electric fields are concentrated along the radiating edges of the patch this is where the meta-atoms will have most effect. Also the majority of the magnetic fields are concentrated at the patch centre, thus the diamagnetic effects of the meta-atom will have little effect on the magnetic fields. The size of the cubic meta-atoms used are $1.25 \mathrm{~mm}$ in all directions with a cube unit cell of $2 \mathrm{~mm}$ in all directions. By using the theory presented by Sihvola the effec-tive relative permittivity of the metallic cube metamaterial is 5.9 [2]. This value agrees with a CST simulation of the meta-atom unit cell in a plane wave which gives the values 5.9 for relative permittivity and 0.67 for the relative permeability. As a reference point, following from previous work [1], the same antenna geometry is also simulated on an artificial dielectric substrate with the same sized meta-atoms and unit cells as pre-viously stated.

In all proposed cases the antenna width, antenna height and substrate thickness remain the same. However, the inset feed distance $\left(Y_{0}\right)$ differs to ensure that in all cases the input imped-ance to the antenna is close to $50 \mathrm{ohm}$. The feeding transmis-sion line width $(W)$ is $5.3 \mathrm{~mm}$ for the conventional patch but $2.2 \mathrm{~mm}$ for the patch on the artificial dielectric substrate. As the meta-atoms provide extra dielectric loading the line width is reduced to maintain a $50 \mathrm{Ohm}$ characteristic impedance for the transmission line. Table. 1 shows the varying inset distances for the patch antennas along with their resonant frequencies, radiation efficiencies and their fractional bandwidths. 


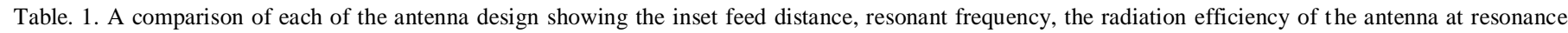
and the fractional bandwidth.

\begin{tabular}{|c|c|c|c|c|}
\hline Patch variation & Inset distance & Resonant frequency & $\begin{array}{c}\text { Radiation efficiency } \\
\text { at resonance }\end{array}$ & Fractional bandwidth \\
\hline Conventional patch & $11 \mathrm{~mm}$ & $2.51 \mathrm{GHz}$ & $69 \%$ & $20.0 \%$ \\
\hline Artificial dielectric substrate & $12 \mathrm{~mm}$ & $2.04 \mathrm{GHz}$ & $51 \%$ & $13.6 \%$ \\
\hline Localised meta-atoms & $13.5 \mathrm{~mm}$ & $2.05 \mathrm{GHz}$ & $62 \%$ & $17.1 \%$ \\
\hline
\end{tabular}

\section{Results}

The antennas were simulated in CST and the reflection coefficients are plotted in Fig. 2. As can be seen, there is a definite advantage to incorporating meta-atoms into the substrate in terms of miniaturisation. By using a heterogeneous artificial dielectric substrate, the resonant frequency has decreased from $2.51 \mathrm{GHz}$ to $2.04 \mathrm{GHz}$. A similar result can be achieved by only placing meta-atoms underneath the radiating edges of the patch. By doing this the miniaturisation benefits can be maintained whilst preserving a high efficiency.

The conventional patch has the largest fractional bandwidth, when comparing the localised meta-atom patch and the artificial dielectric patch the former has a higher fractional bandwidth. Intelligently placing the meta-atoms has also had a lower impact on the fractional bandwidth.

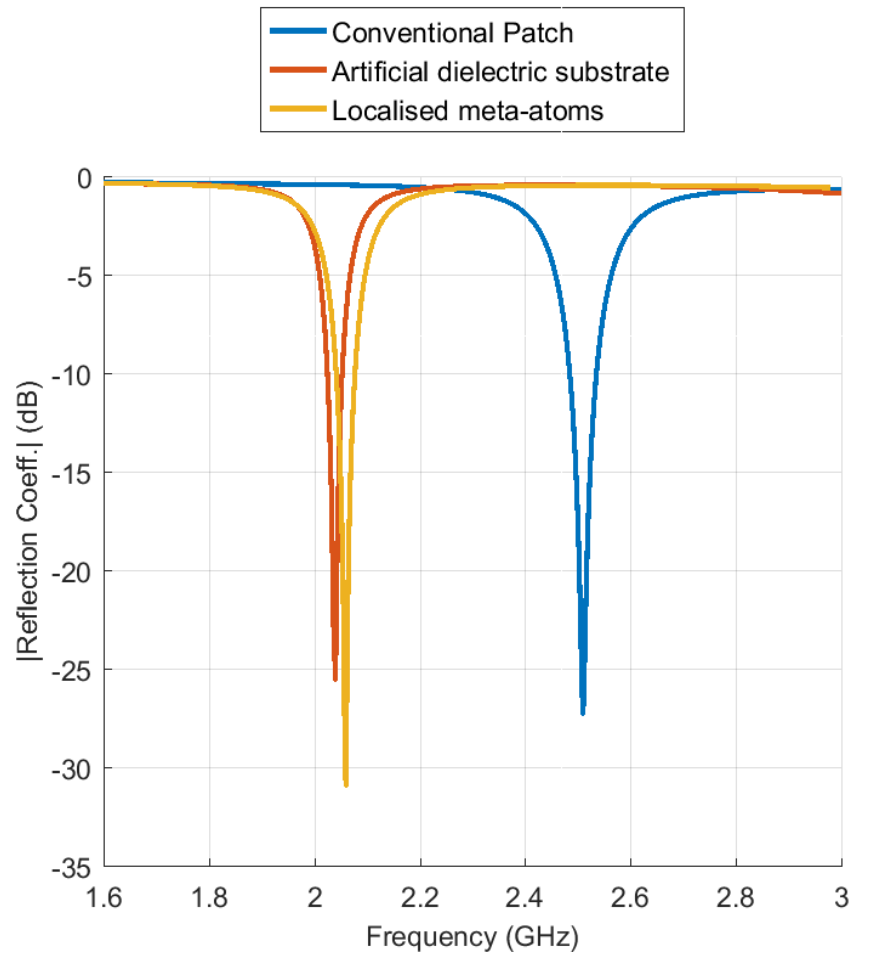

Fig. 2. Simulated reflection coefficient of the conventional patch antenna, the patch antenna on an artificial dielectric and the patch with meta-atoms placed at the radiating edges.

\section{Conclusions}

As has been shown meta-atoms can be used to electrically increase the size of a patch antenna resulting in miniaturisation properties. However, by intelligently placing the meta-atoms in critical locations, instead of using an artificial dielectric substrate, the radiation efficiency and fractional bandwidth can be largely maintained. Using less meta-atoms in the antenna design also means that less metal is used, resulting in lighter weight, less complex and less expensive design to manufacture when compared to artificial dielectric substrates.

\section{Acknowledgments}

This work was supported by the UK Engineering and Physical Sciences Research Council (EPSRC) and is part of the SYMETA project (https://www.symeta.co.uk/). Grant reference code: EP/N010493/1.

\section{References}

[1] C. C. Njoku, W. G. Whittow, and (J)Yiannis C Vardaxoglou, "Antennas on quasi synthetic media," Appl. Phys. A, vol. 115, no. 2, pp. 605-611, May 2014.

[2] S. S. Bukhari and W. G. Whittow, "Heterogeneous Substrate Microstrip Antenna with Enhanced Bandwidth," Loughbrgh. Antennas \{\&\} Propag. Conf., 2013.

[3] L. Lewin, "The electrical constants of a material loaded with spherical particles," Proc. IEE, vol. 94, pp. 65-68, 1947.

[4] A. Sihvola, Electromagnetic Mixing Formulas and Applications. London: IET, 1999.

[5] C. A. Balanis, Antenna theory. Analysis and design, 3rd ed. Hoboken: John Wiley \& Sons, 2005.

[6] S. Zhang, W. Whittow, and J. (Yiannis) C. Vardaxoglou, "Additively manufactured artificial materials with metallic meta-atoms," IET Microwaves, Antennas Propag., vol. 11, no. 14, pp. 1955-1961, Nov. 2017. 